\title{
ESTUDIO DE LAS ACCIONES COMBINADAS A LA OFENSIVA EN LOS FUTBOLISTAS DE LA UNIVERSIDAD DE PAMPLONA.
}

Caicedo. P, Sergio Andrés ${ }^{1}$, Vera. R, José Luis ${ }^{2}$, Ortega. P, Arles Javier ${ }^{3}$

${ }^{1}$ Licenciado en Educación Física Recreación y Deportes. Docente Centro de Bienestar Universitario. Universidad de Pamplona

sergio.caicedo@unipamplona.edu.co

2 Doctor en Ciencias de la Cultura Física. Docente área Educación física y deporte. Universidad del Valle

Vera.jose@corrreounivalle.edu.co

${ }^{3}$ Doctor en Ciencias de la Cultura Física. Docente programa Educación física recreación y deportes. Universidad de Pamplona

arlesjavierortegap@hotmail.com

\section{RESUMEN}

Esta investigación es un estudio descriptivo realizado con la selección masculina de fútbol de la Universidad de Pamplona, buscando analizar su rendimiento durante la fase zonal y regional de los juegos ASCUN deportes 2014, para esto se diseñó e implementó un protocolo de observación de acciones combinadas a la ofensiva que involucra variables determinantes como el inicio y finalización de cada jugada, el tipo de A.B.P. (Acción a balón parado), el rendimiento, tipo de jugada, zona de influencia, el tiempo y la preponderancia numérica, herramienta que fue sometida a juicio de expertos para su validación; este análisis tuvo como fin identificar fortalezas y debilidades a nivel técnico - táctico, en la ejecución de jugadas que limitan el rendimiento de los jugadores en la competencia.

PALABRAS CLAVES: Protocolo, acciones combinadas, ofensiva.

\section{MIXED ATTACK ACTIONS STUDY ON THE PAMPLONA'S UNIVERSITY FOOTBALL PLAYERS.}

\section{ABSTRACT}

This research is a descriptive study conducted by the Pamplona University Football Team, aiming to analyze their performance during the zone and regional stage of ASCUN Sports 2014. In order to accomplish this, we designed and applied an observation protocol of mixed actions on the attack that involves critical variables like the start and the end of each play, ABP type (stopped ball Action), performance, kind of play, area of influence, time and numeric advantage. This tool was reviewed by experts to validade it. This analysis had as aim to identify strengths and weaknesses at the technic and tactical level, in the execution of plays that limit the players performance on the tournament.

KEY WORDS: Protocol, mixed actions, offensive. 


\section{INTRODUCCIÓN}

En la actualidad, el fútbol sigue siendo un fenómeno social de gran importancia debido al aumento progresivo de competiciones importantes, esto nos obliga a profundizar en el entrenamiento contemporáneo, a indagar y adoptar nuevas tendencias, apuntando a un trabajo individualizado que guarde relación con resultados que ya han sido presentados; estos factores traen consigo un perfeccionamiento en el abordaje de los componentes técnico tácticos del fútbol, de manera que dentro de las herramientas fundamentales para desarrollar, optimizar y dar lucidez al deportista en los procesos de entrenamiento, se deben priorizar los elementos estratégicos de las acciones tácticas a la ofensiva, teniendo en cuenta la importancia de la formación de los jugadores a la hora de responder a las expectativas y exigencias que plantea el fútbol competitivo actual; así mismo, es necesario emprender estudios orientados a los problemas y dificultades tácticas de los futbolistas, con el fin de establecer e implementar herramientas, métodos y procedimientos que coadyuven en el perfeccionamiento de las capacidades resolutivas de situaciones de juego.

Partiendo de lo anterior, el proceso ofensivo debe ser una progresión lógica y ordenada, donde predominen los principios (penetración, cobertura ofensiva, movilidad, espacio, ataque y contraataque) y sub principios ofensivos del fútbol, desequilibrando la estructura adversaria, generando un alto grado de incertidumbre al rival y buscando vulnerar el arco contrario con buena velocidad, progresión y dinámica de juego, apuntando a la superioridad 0 igualdad numérica.

Este estudio surge de la necesidad de plantear estrategias que permitan mejorar el rendimiento del equipo de fútbol de la Universidad de Pamplona, Norte de Santander, Colombia. Después de un periodo de observación y análisis, fue posible detectar falencias al momento de realizar una acción de juego a la ofensiva donde predominan características como: poca agilidad para brindar apoyo, crear y aprovechar espacios libres; insuficiencia en cambios de orientación, poco desdoblamiento, creación nula de pequeñas sociedades en el momento de realizar una pared, carencia de orden táctico en progresión o secuencia ofensiva, baja velocidad, amplitud y profundidad, además de una inadecuada conservación del balón. Así mismo, desde el punto de vista táctico prevalece la organización defensiva, lo que minimiza las acciones combinadas a la ofensiva y hace baja la identidad de juego definida, limitando al jugador en desarrollo creativo, anulando esa magia propia e innata de solución a la ofensiva, dando paso a un juego repetitivo y tosco que limita los procesos de progresión.

Es poco el material bibliográfico especializado de autores y entrenadores nacionales que aborden las acciones tácticas a la ofensiva, y permitan realizar un trabajo personalizado y específico en el que se aprecie este componente desde una perspectiva diferente; como estructura general de planificación se realizan trabajos de recuperación un día después de la competencia, entrenamiento de fuerza y resistencia, espacios reducidos, ejercicios integrados, trabajo y automatización en sistemas defensivos con las correcciones del juego anterior, se finaliza con trabajos de definición, acciones a balón parado y estiramientos fuertes para afrontar el siguiente partido. Lo anterior determina la importancia de diseñar estructuras de planificación que fortalezcan las acciones 
tácticas a la ofensiva permitiendo el aprendizaje, control y evaluación del mismo.

\section{Referencias Bibliográficas para la} Fundamentación Teórica.

En cuanto a estudios realizados se encuentran investigaciones en el ámbito nacional e Internacional que se acercan y aportan fundamentos teóricos con relación a las acciones combinadas a la ofensiva, lo que demuestra el interés de varios estudiosos del tema como: (Bangsbo J, Peitersen B, 2003), López (2013), (López López J. , 2003), (Bayer, 1986), (Gréhaigne, 2001), (Mercé, J., Ródenas, L. T., \& Domenech, C. , 2009).

En la literatura especializada tanto de libros como de investigaciones, se proponen aspectos generales importantes como: (López López, 2013) quien en su libro Bases teórico prácticas del entrenamiento especifico del fútbol, plantea una metodología clara, y una caracterización del entrenamiento táctico, aportando a esta investigación la importancia de la organización táctica en la que considera como metodología idónea los siguientes factores: Características del deporte, Características del modelo de juego y estructura del fútbol. Este coctel metodológico del entrenamiento debe tener en cuenta algunos aspectos para el desarrollo de su organización táctica, así:

Momentos del juego: fase ofensiva, transición defensa-ataque, fundamentos, principios y subprincipios, secuencia ofensiva, fases y situaciones del juego. Así mismo, principios tácticos como: penetración, cobertura ofensiva, movilidad, espacio, profundidad, amplitud, progresión y una buena velocidad para circular el balón, deben ser tenidos en cuenta para aplicar correctamente el juego ofensivo.

Por decisión del entrenador, como planificación específica para el presente trabajo se empleó el sistema 1-4-2-1-3, lo anterior con base en las características que observaba en sus jugadores. Realizar un proceso estructurado a la ofensiva considera tener funciones tácticas específicas dentro de un orden lógico como: Transitar el balón de un lado a otro con velocidad y precisión, pases cortos y a ras de piso.

Movimientos pendulares entre líneas para dar salidas en corto, evitando "rifar el balón" Realizar desdobles sobrepasando algunas líneas defensivas sin posesión del balón.

Conservar una distancia adecuada entre líneas en posesión de balón, donde se ocupen y aprovechen los espacios libres.

Mantener un equilibrio ofensivo evitando la inferioridad numérica.

Formación de triángulos para facilitar la circulación del balón.

Desmarques de apoyo y ruptura constantes en campo rival.

Aprovechamiento de los laterales para dar amplitud y profundidad.

Presión de las líneas defensivas adversarias, para producir el error.

Construcción de juego y control en los tiempos de juego.

Teniendo en cuenta lo anterior, López López (2003) define el juego colectivo ofensivo como el "conjunto de acciones individuales y colectivas realizadas por los jugadores del equipo que se encuentra en posesión del balón con el fin de desplegarse y desarrollar sus funciones ofensivas con la máxima eficacia posible".

Por su parte en cuanto a los principios tácticos del juego ofensivo en el fútbol, Bayer (1986) afirma que los principios del juego "representan la fuente de la acción, definen las propiedades invariables sobre las cuales se realizará la estructura fundamental del desarrollo de los acontecimientos".

De esta forma existen tres principios fundamentales en el juego colectivo ofensivo en fútbol:

- Conservación de la posesión del balón: No es un objetivo en sí mismo ya que la posesión del balón no garantiza la progresión ni la finalización, pero ante la incapacidad de progresar es fundamental mantener la posesión del balón. 


\section{- Progresión hacia la portería} contraria: A parte de mantener la posesión del balón es imprescindible superar y desequilibrar la organización defensiva del equipo rival para poder llegar a zonas óptimas para finalizar.

- Finalizar en la portería contraria: Si el juego ofensivo del equipo ha conseguido llegar a zonas cercanas y óptimas para el remate en portería, la finalización es lo que nos dará posibilidades de éxito en el fútbol.

Grehaigne (2001) afirma que existen dos tipos de inicio para el juego colectivo ofensivo:

> Fase estática: Son todas aquellas acciones que se inician a través de un saque (saque de banda, de centro, de esquina, de penalti 0 de falta).

> Fase dinámica: Son todas aquellas acciones que se inician a través de una recuperación del balón.

Existen producciones más complejas que aportan antecedentes teóricos dentro de los que se pueden resaltar estudios realizados en España, donde un trabajo de investigación consistente en un análisis observacional de las transiciones ofensivas realizadas por los mejores equipos nacionales que disputaron la Eurocopa de Austria-Suiza 2008, tratando de identificar aquellas que son más eficaces. Han sido analizados 7 partidos, registrando 743 transiciones ofensivas y se ha elaborado un instrumento de observación que combina los formatos de campo con los sistemas de categorías (Casal Sanjurjo, 2011).

El estudio de (López Bueno, 2007) sobre el análisis de la táctica grupal ofensiva de los equipos participantes en el campeonato mundial de fútbol de Alemania 2006, deja clara la gran repercusión social que tiene el fútbol, deporte objeto de dicho análisis el cual no podía ser indiferente al ámbito científico, éste, buscó analizar el juego desde su propia esencia, que no es otra que la táctica, además, pretende hacerlo con un enfoque comprensivo, tratando de interpretar de una manera razonable los datos obtenidos de las observaciones de los partidos.

Por su parte, (Sánchez Latorre David Luis \& Jiménez Maroto Victoria B., 2001), en su estudio Construcción táctica de un sistema de juego en fútbol. $2^{a}$ parte: desarrollo de las fases del juego, estrategias a balón parado y situaciones de enseñanza aprendizaje, nos aportan información acerca de la construcción táctica del sistema 1-3-43 en fútbol. Son indicaciones relacionadas con las ideas propias entorno al trabajo dentro de nuestro contexto futbolístico. Se detallan las diferentes fases del juego (repliegue, defensa, ataque, contraataque, etc.) y sus posibles modificaciones. Por otro lado se atiende a ejemplos de diferentes estrategias que podrían desarrollarse a balón parado y a situaciones de enseñanza propias de medios colectivos básicos y de la fase de ataque desarrollados en la construcción táctica del sistema de juego. Otro estudio realizado sobre análisis táctico comparativo entre un equipo de fútbol de alto rendimiento frente a otro de iniciación deportiva, fue realizado por (López De La Torre, 2014), quien aporta que en el mundo del deporte, en concreto del fútbol, cada jornada es más difícil ganar un partido y por ello, más difícil afrontar los partidos para disputarlo y sacar una victoria. Nos encontramos con una progresiva profesionalización que el fútbol está experimentado en todos sus ámbitos. Para ello, existe un nuevo concepto para facilitar esta visión del fútbol, y es la de scouting o analista técnico-táctico, que trata de conocer las fortalezas y debilidades del rival al cual se va enfrentar.

Un estudio realizado en Cuba por (Jorge Pensado Delgado \& Leonel Rusell González, 2014) aportan investigaciones sobre consideraciones para el estudio del desempeño táctico en el deporte, donde analizan aspectos relacionados con el componente táctico en el deporte de manera general y de la táctica ofensiva en particular, tomando como referencia al deporte Baloncesto para su estudio. 
Además, se refieren algunos de los antecedentes más importantes sobre su evaluación y se abordan las exigencias y manifestaciones de procesos psicológicos que inciden en este componente.

El estudio realizado por (Coto, L. Oroceno, M. Castillo, S. \& Heredia H, 2013)acerca de un sistema de indicadores y ejercicios para perfeccionar la táctica ofensiva en el equipo juvenil masculino de hockey sobre césped de la provincia Santiago de Cuba, manifiesta que se emplearon métodos del nivel teórico: análisis-síntesis, inductivodeductivo; del nivel empírico: la observación a diferentes topes y entrenamientos, y técnicas como: la entrevista a entrenadores y especialistas, encuesta a atletas y test de relaciones interpersonales. Lo novedoso del aporte se precisa en la posibilidad de aplicar creativamente las fases de la preparación táctica y los principios tácticos ofensivos al sistema de indicadores y ejercicios propuesto, que al ser puesto en manos de atletas, entrenadores y especialistas se constituye en una herramienta de trabajo valiosa encaminada a obtener los resultados esperados. Los métodos y técnicas empleados antes y después de la implementación parcial del aporte, permitieron corroborar la efectividad de la propuesta, el perfeccionamiento y la combinación de las acciones tácticas ofensivas: individuales, grupales $y$ colectivas.

Sobre la base de los argumentos y criterios anteriormente expuestos se ha llegado a precisar como problema científico lo siguiente:

¿Cómo determinar las acciones combinadas a la ofensiva en el equipo de fútbol de la Universidad de Pamplona en los Juegos ASCUN deportes Zonales, Regionales 2014 ?

\section{DESARROLLO METODOLÓGICO}

Este artículo de tipo investigativo descriptivo transversal se constituye en la primera etapa o fase diagnóstica del proyecto de investigación y sintetiza el análisis de los resultados obtenidos a través de la aplicación del protocolo de observación cuyo principal instrumento fue la videografía realizada en cada una de las participaciones de la selección de fútbol de la Universidad de Pamplona en los juegos ASCUN Deportes 2014.

Con esta herramienta de evaluación se buscó determinar y cuantificar el rendimiento de los jugadores en las acciones combinadas a la ofensiva, lo que permitió identificar debilidades y fortalezas a nivel técnico - táctico, resultados que se establecen como línea base para el desarrollo del proyecto general.

\section{DISEÑO DE LA INVESTIGACIÓN}

La presente investigación estuvo encaminada al diseño e implementación de un protocolo de observación como herramienta diagnóstica para el rendimiento de las acciones combinadas a la ofensiva en los jugadores de la selección de fútbol de la Universidad de Pamplona.

Para este abordaje científico se utilizaron los siguientes métodos y técnicas

Analítico Sintético: fue utilizado durante la documentación científica, la sustentación teórica y la consulta a especialistas y expertos

Histórico Lógico: este método estuvo presente en la investigación desde la argumentación que antecedió la problemática abordada, así como en el soporte de resultados cuantitativos obtenidos con anterioridad y su incidencia en resultados actuales.

Observación: el grupo objeto de esta investigación fue estudiado en su propio contexto, en condiciones concretas de competencia con el fin de analizar el rendimiento de los jugadores en las acciones combinadas a la ofensiva.

Medición: fue utilizada para el registro de los indicadores cuantitativos respecto a la cantidad de jugadas y tiempo parcial de ejecución de las acciones combinadas a la ofensiva, discriminadas en criterios así: Tipo de Acción, Tipo de A.B.P. Rendimiento, Tipo de Jugada, Zona de influencia y Preponderancia Numérica 


\section{Selección y descripción de los participantes:}

Para la implementación del protocolo de observación de las acciones combinadas a la ofensiva en fútbol descrito anteriormente, se trabajó con la selección de la Universidad de Pamplona, compuesta en su totalidad por 25 jugadores, sin embargo, esta investigación tuvo en cuenta solo 18 de ellos, los cuales participaron durante la fase zonal y regional de los juegos ASCUN Deportes 2014, realizados en la ciudad de Cúcuta y Bucaramanga respectivamente.

Los resultados obtenidos con la aplicación de este protocolo son producto del análisis de un total de 6 encuentros, 3 de la fase zonal y 3 de la regional donde la Unipamplona enfrentó los seleccionados de instituciones como Universidad Libre, Universidad Francisco de Paula Santander (Cúcuta), Universidad Francisco de Paula Santander (Ocaña), Universidad de los llanos, Universidad Pedagógica y Tecnológica de Colombia y la Universidad Industrial de Santander.

\section{INSTRUMENTOS}

Los partidos fueron grabados con una cámara de video Panasonic modelo NV65300 , con apoyo en trípode y ubicada en el cetro de la gradería con el fin de realizar planos generales que permitieran seguir de manera completa el desarrollo de las jugadas; las grabaciones fueron exportadas al sistema de medición Simi Motion que permite el procesamiento de imágenes y el análisis de movimiento.

Por decisión del entrenador, como planificación específica para el presente trabajo, se empleó el sistema 1-4-2-1-3, lo anterior con base en las características que observaba en sus jugadores.

Para el análisis de las acciones combinadas a la ofensiva en esta investigación, se desarrolló el siguiente protocolo de observación el cual fue sometido a criterio de expertos y su respectivo pilotaje.
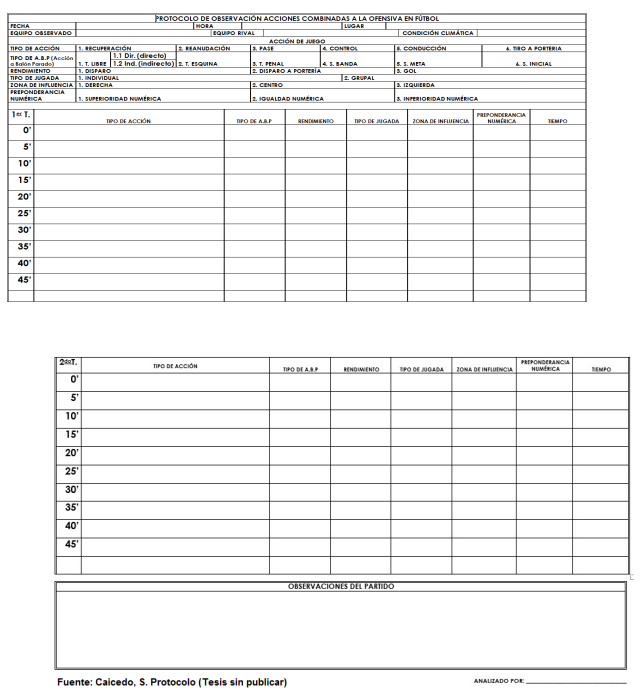

Este protocolo se evalúa a través de videografía; con registros realizados por un mismo evaluador. La observación y registro comienza una vez el observador se familiariza con la herramienta, sentado frente al televisor realiza un análisis depurado de cada acción de juego que presenta la competencia, este protocolo incluye factores determinantes como el inicio y finalización de cada jugada, el tipo de A.B.P. (Acción a balón parado), el rendimiento, tipo de jugada, zona de influencia, el tiempo y la preponderancia numérica; elementos que contribuyen en el desarrollo de la observación de diferentes momentos de la competencia en los que encontramos: fase ofensiva, transición defensa-ataque, fundamentos, principios y subprincipios, secuencia ofensiva, fases y situaciones del juego. Así mismo, principios tácticos como: penetración, cobertura ofensiva, movilidad, espacio, profundidad, amplitud, progresión y una buena velocidad para circular el balón, son aspectos que deben ser tenidos en cuenta para lograr un correcto juego ofensivo. Se realizaron observaciones previas, las cuales fueron tomadas como entrenamiento, al finalizar este proceso el observador realizó la codificación de los datos en dos espacios diferentes dejando como intervalo 8 días entre un registro y otro, en el cual se encuentra similitud en los resultados.

La herramienta presentada anteriormente está conformada por 7 criterios, cada uno 
de ellos con sus respectivas categorías: Tipo de Acción: Recuperación, Reanudación, Pase, Control, Conducción, Tiro a portería. Tipo de A.B.P. (Acción a balón parado): Tiro libre (directo e indirecto), Tiro de Esquina, Tiro Penal, Saque de Banda, Saque de Meta, Saque Inicial. Rendimiento: Disparo, Disparo a Portería, Gol. Tipo de Jugada: Individual, Grupal. Zona de influencia: Derecha, Centro, Izquierda. Preponderancia Numérica: Superioridad, Igualdad, Inferioridad numérica. Tiempo. Cabe aclarar que la unidad de observación se realiza desde que se toma contacto del balón ya sea individual o grupal hasta la pérdida del mismo. Cada secuencia o transición ofensiva se registró en una categoría de los criterios expuestos.

\section{RESULTADOS}

\section{Gráfica 1}

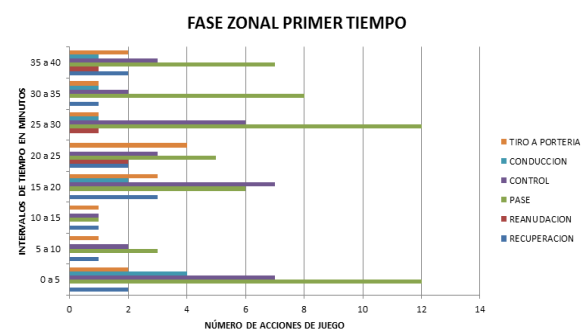

En el análisis de la gráfica No 1 que corresponde al primer tiempo de los partidos de la fase zonal, es evidente la importancia del juego colectivo ofensivo que ha sido definido por López López (2003) como el "conjunto de acciones individuales y colectivas realizadas por los jugadores del equipo que se encuentra en posesión del balón con el fin de desplegarse y desarrollar sus funciones ofensivas con la máxima eficacia posible". en el primer tiempo de esta fase se observó que en los intervalos de 0 a 5 y de 25 a 30 minutos la acción que predominó fue el pase con 12 ejecuciones, así mismo, a nivel grupal esta acción se ejecutó un total de 54 veces, por otra parte, se realizaron 15 tiros a portería con predominancia en el intervalo de 20 a 25 minutos, 4 ejecuciones; las acciones de control se llevaron a cabo principalmente en los periodos de tiempo de 0 a 5 y de 15 a 20 minutos, solo 4 reanudaciones se desarrollaron en el primer tiempo de la fase zonal.

\section{Gráfica 2}

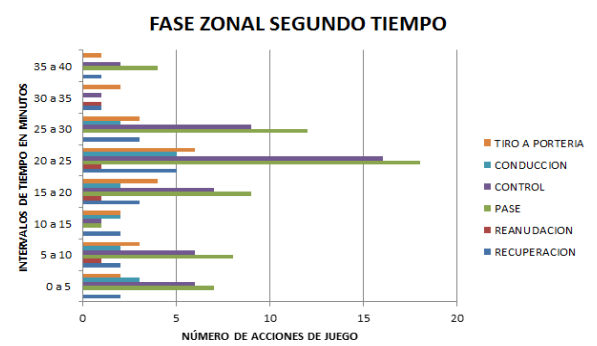

Así como en la gráfica № 1 , en ésta podemos observar que en el segundo tiempo de los partidos de la fase zonal con un total de 59 ejecuciones el pase fue la acción que predominó, de la misma se llevaron a cabo 18 en el intervalo de 20 a 25 minutos, por otra parte es evidente como las acciones de control aumentaron con respecto a las ejecutadas en el primer tiempo de esta fase, se realizaron 16 entre el minuto 20 y el 25 y un total de 48 , así mismo 23 tiros a portería fueron ejecutados predominando igual que en las acciones anteriores en el intervalo de 20 a 25 minutos donde se llevaron a cabo 6 lanzamientos, en este mismo periodo de tiempo 5 acciones de conducción fueron realizadas y un total de 16; de lo anterior podemos inferir que entre el minuto 20 y el 25 del segundo tiempo de la fase zonal, este partido tuvo la mayor frecuencia en ejecución de acciones.

\section{Gráfica 3}

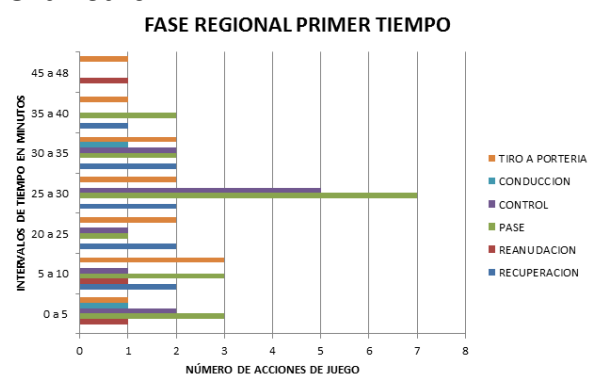

Como lo muestra la gráfica No 3 las acciones como tiro a portería, recuperación, control y pase, tuvieron una frecuencia de 2 ejecuciones por intervalo de tiempo, siendo superiores el pase y el control en el periodo de 25 a 30 minutos con 7 y 5 ejecuciones respectivamente, 3 tiros a portería se realizaron entre el minuto 5 y el 10, 9 acciones de recuperación y 11 de control fueron ejecutadas durante el primer tiempo 
de los partidos de la fase regional, mientras que 18 pases y 12 tiros a portería se llevaron a cabo.

\section{Gráfica 4}

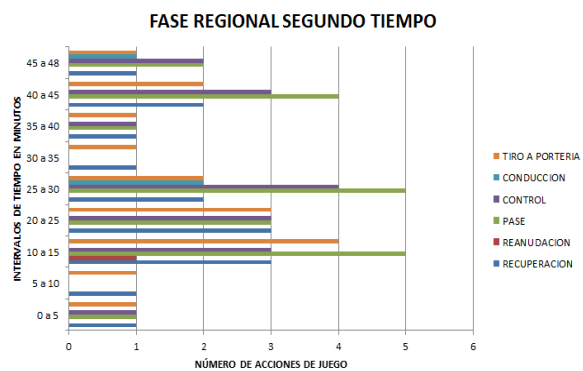

En comparación con la gráfica No 3 es evidente la superioridad numérica en ejecución de acciones, sin embargo, igual que en el primer y segundo tiempo de la fase zonal y el primero de la regional, se ratifican los pases realizados como constante que predomina, en esta ocasión con un total de 21, ejecutados principalmente en los intervalos de tiempo de 10 a 15 y de 25 a 30 minutos, así mismo, se llevaron a cabo 17 acciones de control, 15 de recuperación y 16 tiros a portería, 4 de ellos entre el minuto 10 y el minuto 15 , para un total de 73 acciones ejecutadas con las que finalizó el segundo tiempo de la fase regional.

\section{DISCUSIÓN}

Esta investigación de tipo descriptivo empleó como instrumento evaluador el protocolo de observación de acciones tácticas a la ofensiva en fútbol, planteado y analizado con anterioridad en el presente documento. Evaluar el rendimiento de los jugadores es un común denominador entre las diferentes disciplinas de conjunto, identificar fortalezas y debilidades permite replantear el esquema de juego ajustándolo a las necesidades de los equipos, así mismo, cuantificar el rendimiento durante los partidos facilita evaluar el trabajo realizado previamente y el impacto de las estrategias implementadas.

Indagando en el tema se encuentra un protocolo de observación del comportamiento táctico en practicantes de baloncesto diseñado por (Martínez, D. Martínez, R \& Mapolón, J, 2011) con el que se observan varias similitudes, pues en él se hace un análisis de variables como posesión y recuperación del balón, orientación y protección del aro, cada uno de ellos se compone de categorías que apuntan a la identificación de jugadas que permiten la cuantificación del rendimiento deportivo en táctica ofensiva.

Las similitudes encontradas con el protocolo ya mencionado evidencian criterios comunes de gran relevancia en ejecución de acciones tácticas ofensivas, así mismo, ratifican la necesidad, posibilidad e importancia de implementar herramientas como esta en diferentes deportes de conjunto, que apoyadas en instrumentos como la videografía facilitan el análisis detallado de criterios determinantes en el rendimiento deportivo y el diseño de estrategias para su mejoramiento.

Teniendo en cuenta lo expuesto por (González Terry, 2004) se infiere que los protocolos de observación comparados en esta discusión se emplean para realizar un registro de frecuencia donde después de la observación se puede obtener la suma de acciones ejecutadas en cada uno de los criterios, lo que posibilita determinar la predominancia de principios ofensivos 0 defensivos en el juego, lo anterior permite replantear a nivel técnico- táctico los procedimientos estratégicos implementados que contribuyan el logro de objetivos trazados.

\section{CONCLUSIONES}

Después de haber analizado el proceso de registro, el protocolo de observación es un instrumento que incluye los elementos de información observable y necesaria, de contenido y precisión para el estudio detallado de las acciones combinadas a la ofensiva, el cual favorece el rendimiento de la selección de fútbol de la Universidad de Pamplona.

Como resultado de la investigación presentada, se puede concluir que el 
instrumento de evaluación es una herramienta útil para el análisis de las acciones combinadas a la ofensiva en fútbol, que permite cuantificar el rendimiento, identificar fortalezas y debilidades con una aplicabilidad en las diversas categorías del fútbol.

\section{REFERENCIAS BIBLIOGRÁFICAS}

Bangsbo J, Peitersen B. (2003). Fútbol, jugar en ataque. Editorial Paidotribo.

Bayer, C. (1986). La enseñanza de los juegos deportivos colectivos: Baloncesto, futbol, balonmano, hockey sobre hierba y sobre hielo, rugby, balonbolea, waterpolo. Hispano Europea.

Casal Sanjurjo, C. A. (2011). Estudio descriptivo de las transiciones en el fútbol. EFDeportes, 1.

Coto, L. Oroceno, M. Castillo, S. \& Heredia H. (2013). sistema de indicadores y ejercicios para perfeccionar la táctica ofensiva en el equipo juvenil masculino de hockey sobre césped de la provincia Santiago de Cuba. EFDeportes, 1.

González Terry, C. (2004). La Investigación Científica en la Actividad Física: su Metodología. Ciudad de la Habana. Cuba: Editorial Deportes.

Gréhaigne, J. (2001). La organización del juego en el fútbol (1st ed). Barcelona: INDE, 2001.

Jorge Pensado Delgado \& Leonel Rusell González. (2014). Consideraciones para el estudio del desempeño táctico en el deporte. EFDeportes, 1.
López Bueno, R. (2007). Análisis de la táctica grupal ofensiva de los equipos participantes en el campeonato mundial de fútbol Alemania 2006. EFDeportes, 1.

López De La Torre, J. L. (2014). Análisis táctico comparativo entre un equipo de fútbol de alto rendimiento frente a otro de iniciación deportiva. EFDeportes, 1.

López López, J. (2003). Fútbol: Fundamentos tácticos ofensivos (1st ed). Wancuelen Editorial Deportiva.

López López, J. (2013). Bases Teóricoprácticas del entrenamiento específico del fútbol. www.entrenarfútbol.es.

López, L. (2013). Modelo táctico del fútbol club Barcelona. Organización táctica ofensiva y defensiva. Propuesta de entrenamiento. www.entrenarfútbol.es.

Martínez, D. Martínez, R \& Mapolón, J. (2011). Protocolo de observación para el control del comportamiento táctico en prácticantes de baloncesto de 9 y 10 años en el municipio Florida. EFDeportes, 1-9.

Mercé, J., Ródenas, L. T., \& Domenech, C. . (2009). Fútbol: Sistema 1.4.2.3.1. Sevilla: Wancuelen.

Sánchez Latorre David Luis \& Jiménez Maroto Victoria B. (2001). Construcción táctica de un sistema de juego en fútbol. $2^{\mathrm{a}}$ parte: desarrollo de las fases del juego, estrategias a balón parado y situaciones de enseñanza aprendizaje. EFDeportes, 1. 